\title{
Modeling and Simulation for a Quadrotor Vehicle With Adaptive Wing
}

\author{
Qunpo Liu \\ School of Electrical Engineering and Automation, Henan Polytechnic University, 2001 Century Avenue \\ Jiaozuo (454003), Henan, P.R. China \\ Hongqi Wang \\ School of Electrical Engineering and Automation, Henan Polytechnic University, 2001 Century Avenue \\ Jiaozuo (454003), Henan, P.R. China \\ Fuzhong Wang \\ School of Electrical Engineering and Automation, Henan Polytechnic University, 2001 Century Avenue \\ Jiaozuo (454003), Henan, P.R. China \\ Jikai Si \\ School of Electrical Engineering and Automation, Henan Polytechnic University, 2001 Century Avenue \\ Jiaozuo (454003), Henan, P.R. China \\ Naohiko Hanajima \\ College of Information and Systems, Muroran Institute of Technology, 27-1 Mizumoto-cho,Hokkaido \\ Muroran-shi, Hokkaido (050-8585), Japan \\ E-mail: lqpny@hpu.edu.cn; wangfzh@hpu.edu.cn, sijikai527@126.com,hana@mondo.mech.muroran-it.ac.jp \\ www.hpu.edu.cn, www.muroran-it.ac.jp
}

\begin{abstract}
Aiming at developing high power efficiency system for quadrotor vehicle during height keeping and long distance flight, a quadrotor vehicle with adaptive wing is proposed. Angle of the wing respect to flying direction can be adjusted adaptively according to flying speed, to reduce energy expenditure of motor. The dynamic model was built up. Rotor lift were analyzed based on the mechanical structure and its flight principle. The simulation is carried out to verify the effectiveness of high power efficiency.

Keywords: Modeling, Quadrotor vehicle, Simulation, Wing.
\end{abstract}

\section{Introduction}

Because of distinct features, such as vertical take-off and landing(VTOL), sharp maneuvering, low cost, simple mechanism, UAVs have attracted more and more interests in a wide range involving rescues, security, inspection, surveillance, aerial photography, mapping, etc. Each individual rotor has a smaller diameter and less kinetic energy than the equivalent single-rotor helicopter. Compared to conventional helicopters, with the large main rotor and tail rotor, the quadrotor is easier to fly, does not have the complex swash plate mechanism and is easier to model and control. The quadrotor has a promising application as commercial, military and academic platforms.

Much research has focused on the Dynamic modeling ${ }^{[1][5]}$, simulation, optimization, controller $\operatorname{design}^{[2]}$, and control algorithm ${ }^{[3][4][5]}$ for quadrotor 
vehicle. However, there are still some problems in practical use, such as energy expenditure during long distance forward flight. By now, lithium battery is used for power supply for quadrotor vehicle. But the weight of the batteries increases with the capacity. At the same time, the energy expenditure is also increases for increased weight and dimension. Due to the conflict between the weight of the batteries (capacity) and flight distance, flight distance is subject to the restriction. However, there are relatively fewer contributions on the energy expenditure during long distance forward flight for a quadrotor. Therefore, how to reduce the energy expenditure during long weight is a problem to solve for the application of quadrotor vehicle.

A quadrotor vehicle with adaptive underbelly wing is proposed. The underbelly wing at certain angles is expected to obtain a horizontal direction of thrust. Advantageously, this may result in a reduction of dip angle for quadrotor body and also can resulting in lower airflow resistance and lower downward force on quadrotor body. And finally, it can eventually reduce energy expenditure during long distance forward flight.

\section{Dynamic modeling}

This section presents a simple model of the quadrotor dynamics according to reference [5] and [6].

As shown in Fig.1, the quadrotor possesses a simple and symmetrical structure with four rotors and can be controlled by the rotational speed of the four rotors.

For the sake of convenience, $T_{l}$ is denoted as the head of the rotorcraft. Meanwhile rotor $T_{3}$ is the tail. i.e., $\boldsymbol{T}_{1}, \boldsymbol{T}_{2}, \boldsymbol{T}_{3}$ and $\boldsymbol{T}_{4}$ is named front, right, rear, left rotor respectively. Define the following body coordinate system $\boldsymbol{x}_{\boldsymbol{b}}, \boldsymbol{y}_{\boldsymbol{b}}, \boldsymbol{z}_{b}$ : the original point $\left(\boldsymbol{O}_{\boldsymbol{b}}\right)$ is the center of gravity; $\boldsymbol{x}_{\boldsymbol{b}}$ is from b $\boldsymbol{O}_{\boldsymbol{b}}$ the head of the quadrotor; $\boldsymbol{y}_{\boldsymbol{b}}$ is from $\boldsymbol{O}_{b}$ to the right side of the quadrotor; and $\boldsymbol{z}_{b}$ follows the law of right hand.

The notation for the quadrotor model is shown in Fig. 2. Define the following inputs to model the quadrotor: $\boldsymbol{F}_{\text {Thrust }}$ is the thrust generated by four motors; $F_{Z}$ is the the vertical upward thrust force on the quadrotor body; $F_{b f}$ is the air resistance on the body; $F_{w a}$ is the left force on the wing; $F_{W f}$ is the air resistance on the wing; $\theta$ is the pitch of the quadrotor body; $\boldsymbol{\alpha}$ is the angle between the $\boldsymbol{x}_{\boldsymbol{b}}$ and the wing; $\boldsymbol{\gamma}$ is the angle of

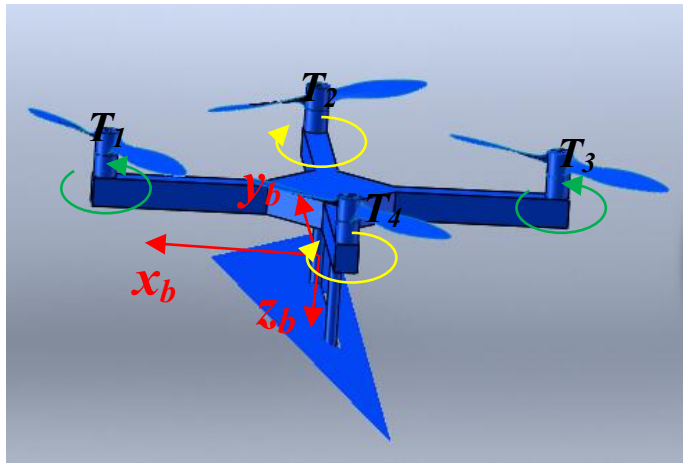

Fig.1 The main structure of a quadrotor

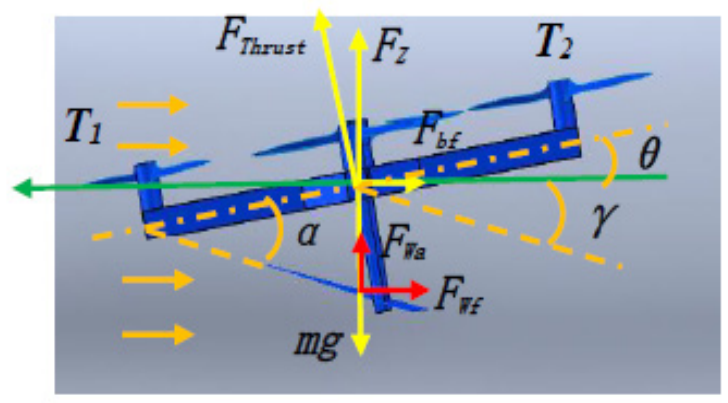

Fig.2 Force modeling of the quadrotor

between the wing and the horizontal flying direction which marked by a line in green. $\boldsymbol{m g}$ is the weight of the quadrotor. All the analysis is on the assumption of steady volume of air flow situation.

The rotor speed is $\boldsymbol{\omega}_{\boldsymbol{i}}$ and the thrust force is an upward vector

$$
F_{\text {Thrust }}=\sum_{i=1}^{4} b \omega_{i}^{2}
$$

in the vehicle's negative $z$-direction, where $b>0$ is the lift constant that depends on the air density, the cube of the rotor blade radius, the number of blades, and the chord length of the blade.

When the quadrotor is flying in a certain speed on certain height, the vertical upward thrust force on the quadrotor body should be remained unchanged.

$$
F_{z}=F_{\text {Thrust }} \cos \theta+F_{w a}-m g
$$

and

$$
F_{w a}=\frac{1}{2} C_{y} \rho_{\infty} V_{\infty} S \cos (\alpha+\theta)
$$


where, $\boldsymbol{C}_{\boldsymbol{y}}$ is wing section lift coefficient; $\boldsymbol{S}$ is the area of the wing; $\boldsymbol{\rho}_{\infty}$ is the air density; $\boldsymbol{V}_{\infty}$ is the steady speed.

$F_{w a}$ is perpendicular to the direction of air flow.

Deviation of the direction of air flow caused by wash velocity can be ignored for low speed.

The force $F^{\prime}{ }_{w f}$ on the quadrotor body generated by the air resistance on the wing $F_{w f}$ is

$$
F_{w f}^{\prime}=F_{w f}+F_{w f} \cos (-\theta) \times d
$$

And it can be counteracted by increasing of $\omega_{3}$ and reducing of $\omega_{1}$.

The air resistance $F_{W f}$ on the wing is

$$
F_{w f}=\frac{1}{2} C_{y}^{\prime} \rho_{\infty} V_{\infty} S \sin (\alpha+\theta)
$$

where, $\boldsymbol{C}_{\boldsymbol{y}}{ }_{\boldsymbol{y}}$ is wing resistance coefficient.

When the quadrotor is fly on same height, the force on the body in vertical direction is

$$
F_{z}=F_{\text {Thrust }} \cos \theta+F_{w a}-m g=0
$$

Therefore, the equation can be obtained using Eq. (3) and (5)

$$
m g-F_{\text {Thrust }} \cos \theta=\frac{1}{2} C_{y} \rho_{\infty} V_{\infty} S \cos (\alpha+\theta)
$$

During the flying, the force on the moving direction is

$$
F_{x}=F_{\text {Thrust }} \sin \theta-F_{w f}=m a_{x}
$$

where, $a_{x}$ is acceration of quadrotor on the moving direction.

When the speed is stable, $a_{x}=0$,

$$
F_{\text {Thrust }} \sin \theta=-\frac{1}{2} C_{y}^{\prime} \rho_{\infty} V_{\infty} S \sin (\alpha+\theta)
$$

In the situation without wing of quadrotor,

$$
F_{\text {Thrust }} \cos \theta=m g
$$

In the situation with a wing of quadrotor, the following equation can be deduced from Eq. (7) and (9)

$$
\therefore F_{\text {Thrust }}=\frac{m g C_{y}^{\prime} \sin (\alpha+\theta)}{C_{y}^{\prime} \cos \theta \sin (\alpha+\theta)-C_{y} \sin \theta \cos (\alpha+\theta)}
$$

Define that

$$
\delta=\frac{C_{y}^{\prime} \sin (\alpha+\theta)}{C_{y}^{\prime} \cos \theta \sin (\alpha+\theta)-C_{y} \sin \theta \cos (\alpha+\theta)}
$$

Therefore, Eq. (11) can be simplified as

$$
F_{\text {Thrust }}=\delta m g
$$

From Eq.(13), it can be draw that the energy expenditure would be deceased if $0 \leq|\delta|<1$.

\section{Simulation results}

To verify whether there are same cases meet requirements, analysis is carried out based on MATLAB platform.

In the Eq.(12), there are two parameters $\boldsymbol{C}_{\boldsymbol{y}}$ and $\boldsymbol{C}_{\boldsymbol{y}}$. Normally, Lift-drag ratio $\boldsymbol{k}$ is used instead. Lift-drag ratio $\boldsymbol{k}$ is relate to wing's angle of attack and can be measured from wind tunnel test:

$$
k=\frac{C_{y}}{C_{y}^{\prime}}
$$

The lift-drag ratio $\boldsymbol{k}$ is simulated by wind tunnel test data in reference [7], as shown in Fig.3. And it is used in the conclusion of $\boldsymbol{\delta}$.

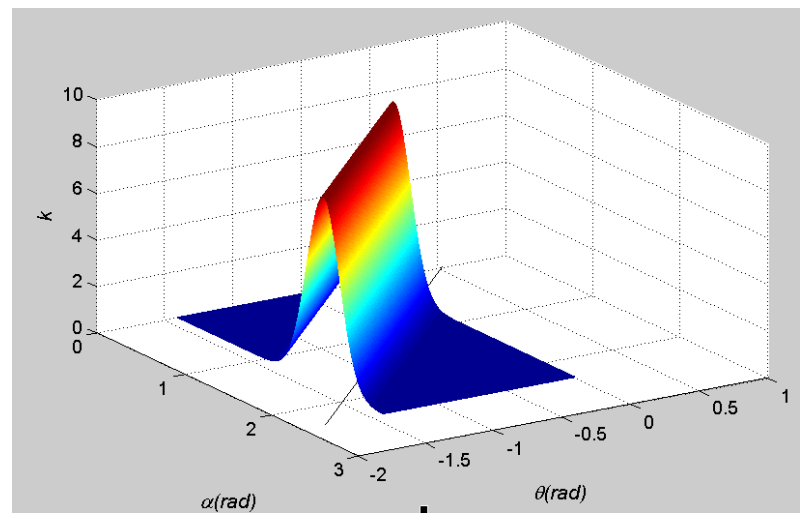

Fig.3 Lift-drag ratio $\boldsymbol{k}$

In the area marked $\mathrm{A} 1$, the results are not valid because it falls outside the allowable range $(\boldsymbol{\alpha}+\boldsymbol{\theta}) \leq \boldsymbol{0}$.

In the area marked A3, the value of $\delta$ is bigger than 1 , which is means more energy needed than quadrotor without wing. 
As shown in Fig.4 and Fig.5, in the area (A2) with color changing from dark blue to dark violet in middle of the figure, the value of $\boldsymbol{\delta}$ is $0 \leq \boldsymbol{\delta} \leq 1$. It means the thrust force is smaller than the gravity of guardrotor. Especially in same point, the thrust force is zero in idea situation. And it also can be draw from the simulation results that the thrust force will increase with $(\boldsymbol{\alpha}+\boldsymbol{\theta})$ increasing. Expecially when $\boldsymbol{\alpha}+\boldsymbol{\theta}>\pi / 6$ (the line as shown in Fig.5), value of $\boldsymbol{\delta}$ increase dramatically, which is due to decrease of the lift-drag ratio $\boldsymbol{k}$. In this case however, $\boldsymbol{\delta}$ and the thrust force $\boldsymbol{F}_{\text {Thrust }}$ will increase very much even $\boldsymbol{\delta}$ is small in the time.

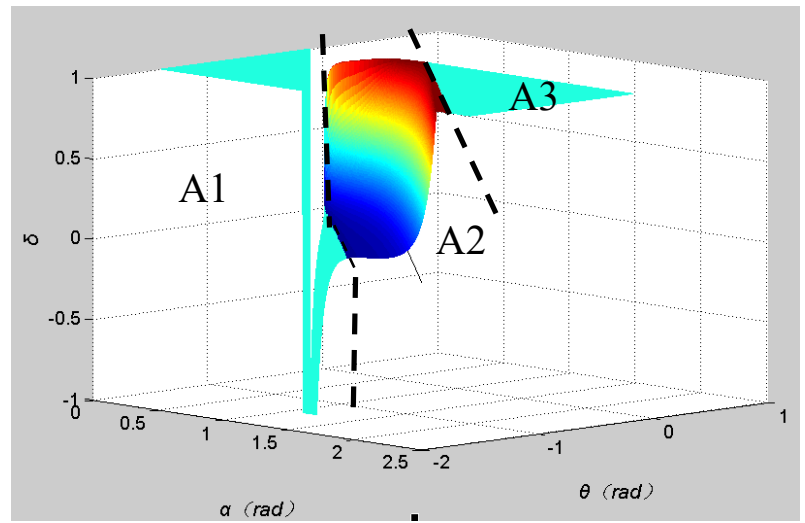

Fig.4 D view of simulation results of $\boldsymbol{\delta}$

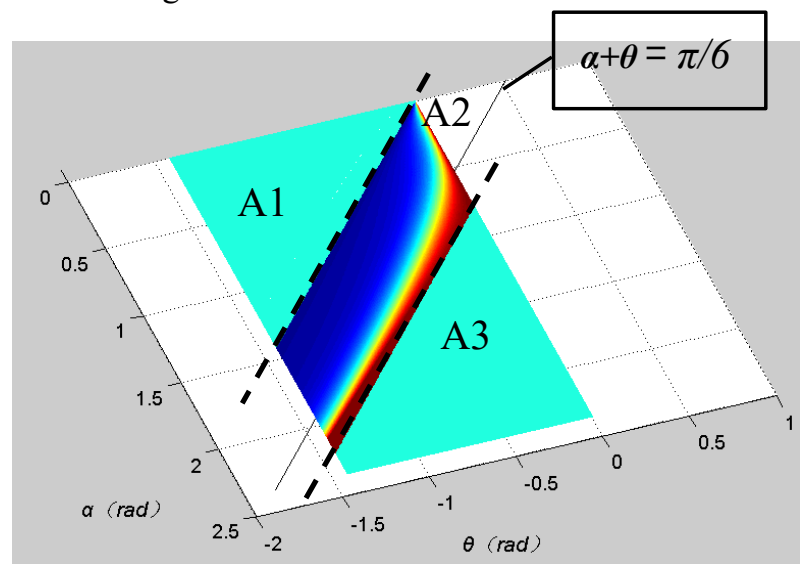

Fig.5 Top view of simulation results of $\boldsymbol{\delta}$

Therefore, the best to reduce energy expenditure is on situation of $0<\boldsymbol{\alpha}+\boldsymbol{\theta}<\pi / 6$. In this case, the thrust force $\boldsymbol{F}_{\text {Thrust }}$ will be stable invariable when $(\boldsymbol{\alpha}+\boldsymbol{\theta})$ does not change. If $\boldsymbol{\theta}$ decreases under influence of air flow, the value of $\boldsymbol{\alpha}$ should increase to keep stable of $\boldsymbol{F}_{\text {Thrust }}$. In this case, $\boldsymbol{F}_{\text {Thrus }^{*} \boldsymbol{c}} \boldsymbol{c o s} \boldsymbol{\theta}$ will increase according to
Eq.(6). The $\boldsymbol{F}_{\text {Thrust }}$ will decrease if the flying velocity $V$ remains constant. Considering Eq.(8), the force on $\mathrm{x}$ direction is

$$
\left|F_{\text {Thrust }} \sin \theta\right|<\left|-\frac{1}{2} C_{y}^{\prime} \rho_{\infty} V_{\infty} S \sin (\alpha+\theta)\right|
$$

Therefore, the flying velocity V should decrease until a new stable equilibrium is reached.

\section{Conclusions}

In this paper, a quadrotor vehicle with adaptive underbelly wing is introduced. A simplified mathematic model is studied firstly. The force modeling was analyzed. Simulation results show that the proposed quadrotor vehicle with adaptive underbelly wing can reduce energy expenditure by arranging the attitude angle of wing within limited range.

\section{Acknowledgements}

Supported by Innovation Scientists and Technicians Troop Construction Projects of Henan Province (Grant No. CXTD2016054)

\section{References}

1. Hai-Nguyen Nguyen,ChangSu Ha,Dongjun Lee, Mechanics, control and internal dynamics of quadrotor tool operation, Automatica, 61 (2015) 289-301

2. Das A, Lewis F, Subbarao K. Backstepping approach for controlling a quadrotor using lagrange form dynamics[J] J Intell Robot Syst., 56: (2009) 127-151.

3. Wei Dong, et ac. A high-performance flight control approach for quadrotors using a modified active disturbance rejection technique, Robotics and Autonomous Systems, 83 (2016) 177-187

4. Juhi Ajmera, V. Sankaranarayanan, point-to-point control of a quadrotor theory and experiment, IFAC-Papers On Line, 49 (1), (2016), 401-406

5. Peter Corke, Robotics, Vision and Control (Springer Verlag, Berlin Heidelberg, 2011).

6. WANG Hong-yu, et ac. Modelling and position tracking control for quadrotor vehicle, Journal of Chinese Inertial Technology, 20(4) (2012) 455-458

7. WANG Bingliang, et ac. Vehicle Aerodynamics (Tsinghua University Press, Beijing, 2013) 